$\mathbb{T}$ periodica polytechnica

Transportation Engineering

$41 / 1(2013) 13,20$

doi: 10.3311/PPtr.7094

http://periodicapolytechnica.org/tr

Creative Commons Attribution (i)

RESEARCH ARTICLE

\section{Electronic stability program with vehicle sideslip estimation}

\author{
Zoltán Hankovszki / Roland Kovács / László Palkovics
}

Received 2012-11-05

\begin{abstract}
The recently used vehicle stability algorithms primary use yaw rate and lateral acceleration as reference signals while vehicle state could be defined by yaw rate and vehicle sideslip angle. Furthermore onboard later acceleration measurement is overlayed with significant disturbances. Approximate enough vehicle sideslip estimation ensures the opportunity of correct vehicle state observation and deducing other important vehicle parameters as corrected lateral acceleration for instance. By using this additional information new vehicle state definitions and interventions are realizable. The front and rear axles' cornering stiffness coefficients could be estimated from the vehicle sideslip angle - after some filtering these signals can show which wheels could be braked. Or new reference models could be set up - these models will be adaptive, not like the currently used empirically defined models. Adaptive means that it considers adhesion coefficients, tyre characteristics and vehicle load at the same time - not as the recently used systems. Predicted vehicle state definition could also be done, which is able to show dangers before they start to develop.
\end{abstract}

\section{Keywords}

vehicle sideslip estimation - cornering stiffness coefficient . adaptive reference model $\cdot$ vehicle stability $\cdot$ active steering · rollover protection $\cdot$ directional control

\section{Acknowledgement}

This work has been partially supported by TRUCKDAS and TÁMOP $421 B$

\section{Zoltán Hankovszki}

Department of Automobiles and Vehicle Manufacturing, Budapest University of Technology and Economics, Stoczek street, H-1111 Budapest, Hungary e-mail: Hankovszki.Zoltan@auto.bme.hu

\section{Roland Kovács}

Knorr-Bremse R\&D Center, Major u. 69, H-1119 Budapest, Hungary e-mail: roland.kovacs@knorr-bremse.com

\section{László Palkovics}

Department of Automobiles and Vehicle Manufacturing, Budapest University of Technology and Economics, Stoczek street, H-1111 Budapest, Hungary e-mail:palko@auto.bme.hu

\section{Introduction}

Active vehicle safety is a timeless and actual problem. The development rate of road traffic and road infrastructure is not equal - former is faster. Economical, sociological and technological conditions [11] result that traffic density is growing. This requires much and much safer vehicles that are able to handle heavier traffic - accurate steering systems, short stopping distances, driver aiding systems, etc. The recently used vehicle stability control software (ESP as electronic stability program) is effective, that's not a question. In the United States, where high centre of gravity (CoG) point sport utility vehicles (SUV) are popular, the rollover risk was reduced by nearly $80 \%$ and the number of fatal crashes was decreased with about $30 \%$ in case of ESP using vehicles [5]. It has to be noticed that even four-wheel-driving could reach hardly any advantage from the viewpoint of fatal accidents [3].

In spite of this recently used ESP systems have significant inaccuracies: their inputs are vehicle velocity from wheel speeds, steering wheel angle, yaw rate and lateral acceleration. From these signals an exact and direct vehicle state definition is not possible - estimations are needed. Estimation of vehicle sideslip angle, and tyre grip abilities, frame roll angle, road bank angle and corrected lateral acceleration for example. These signals could result in more accurate vehicle state definition and interventions. It is important that all of the few mentioned estimations are based on the estimation of vehicle sideslip angle, so the estimation accuracy of this one signal is fundamental. Furthermore nowadays' stability system's setup is difficult, subjective and vehicle state dependent. Latter means that often only one linear reference model is used [3] whose parameters are empirically tuned and it should be suitable for every vehicle condition and it doesn't consider adhesion coefficient or vehicle load.

Based on these facts electronic stability software's development has several opportunities, which could improve the effectiveness of a system like this, but these chances are hard to realize. New sensors or new software are needed. Former often are too expensive for a series product - the costs of a basic ESP sensor set with intervention units and ECU are about two magnitudes lower than that of an optical sideslip angle sensor. The 
other way is to use new software probably with estimation methods. In literature several estimation methods can be found. To mention only one of the lots see [8]. It estimates frame pitch and roll, road bank, vehicle sideslip angle, adhesion coefficient and longitudinal wheel slips [15] for example. Our aim is to develop an estimation method that results in similarly estimated physical quantities but with as low as possible requirements. Latter means that no predefined vehicle properties are considered: no tyre force characteristics, no known vehicle mass and inertia matrix or suspension spring and damping parameters. Essentially we don't want to use any varying information that could define a part of the vehicle's behaviour in a given vehicle condition, to avoid that condition changes (e.g. worn or brand new tires) decrease the effectiveness of our estimation [4]. Furthermore in many cases it is not possible to measure some vehicle parameters - an example is commercial vehicles' significant payload that could be more than double of the vehicle's empty mass.

No matter how but if vehicle sideslip angle is estimated accurately enough ESP could be improved. This paper focuses on these improvements. Active servo motor, sideslip based directional control, and predictive rollover protection [10] will be the topic. With active servo torque a vehicle skid could be handled, or the driver could learn the vehicle's actual capabilities. This teaching can result not just safer but more economical and environment friendly driving that is a huge technical challenge [7]. Directional control is part of ECE 13 [16] and now it is often based on yaw rate control. Instead of this sideslip control could be better: if the sideslip is in a tight range with respect to the longitudinal vehicle plane then an average driver is able to control the vehicle [13]. Moreover there are possible situations that are hidden for a classical yaw control but sideslip control can detect them. By knowing the estimated sideslip value an approximated bicycle model could be set up and with some further steering wheel angle analysis predictions could be taken.

\section{Vehicle state definition}

\subsection{Definition of vehicle dynamics}

First of all vehicle state definition must be laid down. For this vehicle's lateral dynamic should be defined. Basing on pure kinematical behaviors the Ackermann geometry describes vehicle's maneuver during steering but if force effects are considered then frictions will play an important roll [17]. To clear the situation let's start with one of the simplest laws by the Newton-Euler dynamics [1]. Take a mass point in a $2 \mathrm{D}$ plane with a velocity vector: the point has 2 degrees of freedom (DoF) and the velocity has a $v$ magnitude and a $\theta$ direction (1). The base vectors of the plane are $i$ and $j$. The direction is considered with respect to the $i$ vector. Furthermore define an $e_{T}$ tangential vector that is parallel to the velocity's direction (2) and an $e_{N}$ normal vector that is perpendicular to the velocity's direction (3). The acceleration of this mass point could be calculated with partial derivation of the velocity's magnitude and its direction vector (4). If calculations are done than it could be seen that tangen- tial vector's derivative is parallel to normal vector (5) and the acceleration will have a tangential and normal part (6).

$$
\begin{aligned}
\underline{v} & =|\underline{v}| \cdot \underline{e}_{T}=v \cdot \underline{e}_{T} \\
\underline{e}_{T} & =\underline{i} \cdot \cos (\theta)+\underline{j} \cdot \sin (\theta) \\
\underline{e}_{N} & =\underline{j} \cdot \cos (\theta)-\underline{i} \cdot \sin (\theta) \\
\underline{a} & =\frac{d v}{d t} \cdot \underline{e}_{T}+v \cdot \frac{d \underline{e}_{T}}{d t} \\
\frac{d \underline{e}_{T}}{d t} & =\frac{d \theta}{d t} \cdot(\underline{j} \cdot \cos (\theta)-\underline{i} \cdot \sin (\theta)) \\
\underline{a} & =\frac{d v}{d t} \cdot \underline{e}_{T}+v \cdot \frac{d \theta}{d t} \cdot \underline{e}_{N}
\end{aligned}
$$

If a vehicle is given then its body will have a $\psi$ yaw direction too (this is the $3^{\text {rd }}$ DoF) and this direction is not the same as the body's velocity's $\theta$ direction. The difference of them is called as $\beta$ vehicle sideslip angle (7). Longitudinal $v_{X}$ and lateral $v_{Y}$ velocity (8) and longitudinal $a_{X}$ and lateral $a_{Y}$ acceleration (9) could also be defined in the vehicle body's coordinate system that together with the yaw velocity are what sensors can measure.

$$
\begin{aligned}
& \theta=\beta+\psi \Rightarrow \frac{d \theta}{d t}=\frac{d \beta}{d t}+\frac{d \psi}{d t} \\
& {\left[\begin{array}{l}
v_{X} \\
v_{Y}
\end{array}\right] }=\left[\begin{array}{c}
\cos (\beta) \\
\sin (\beta)
\end{array}\right] \cdot v \\
& {\left[\begin{array}{l}
a_{X} \\
a_{Y}
\end{array}\right]=\left[\begin{array}{rr}
\cos (\beta) & -\sin (\beta) \\
\sin (\beta) & \cos (\beta)
\end{array}\right] \cdot\left[\begin{array}{c}
\frac{d v}{d t} \\
v \cdot \frac{d \theta}{d t}
\end{array}\right] }
\end{aligned}
$$

\subsection{Definition of force effects}

By neglecting the longitudinal tyre forces and simplifying the vehicle model the previously defined $3 \mathrm{DoF}$ case could result in a good enough vehicle state definition. Simplifying means that only one tyre is considered in case of an axle (and this tyre will be in the middle of the axle), and only two axles are allowed for a vehicle (if there are more axles then axle group's weighted middle should be considered), so altogether two lateral forces should considered and their attack point is in the vehicle's longitudinal plane. Furthermore linear tyre characteristics are supposed, so $F_{Y}$ tyre force is shown by $(10)$, where $\alpha$ is the tire's sideslip angle and $c$ is the tire's cornering stiffness coefficient. From the previous vehicle dynamics $v_{Y 1}^{v e h}$ front and $v_{Y 2}^{v e h}$ rear virtual tires' lateral velocities from the viewpoint of tires could be seen by (11). Markings $l_{1}$ and $l_{2}$ mark the axle distances' absolute values from $\mathrm{CoG}$, and the dot over $\psi$ marks derivation by time.

$$
\begin{aligned}
F_{Y} & =\alpha \cdot c \\
{\left[\begin{array}{c}
v_{Y 1}^{v e h} \\
v_{Y 2}^{v e h}
\end{array}\right]=} & {\left[\begin{array}{rr}
-1 & -l_{1} \\
-1 & l_{2}
\end{array}\right] \cdot\left[\begin{array}{c}
v_{Y} \\
\dot{\psi}
\end{array}\right] } \\
\alpha_{2}= & \arctan \left(\frac{v_{Y 2}}{v_{X 2}}\right)=\arctan \left(\frac{v_{Y 2}^{v e h}}{v_{X}}\right) \\
& \text { where } \frac{v_{Y 2}}{v_{X}}=-\frac{v_{Y}}{v_{X}}+\frac{\dot{\psi} \cdot l_{2}}{v_{X}}
\end{aligned}
$$




$$
\begin{aligned}
{\left[\begin{array}{l}
v_{X 1} \\
v_{Y 1}
\end{array}\right]=} & {\left[\begin{array}{cc}
\cos \left(\delta_{1}\right) & -\sin \left(\delta_{1}\right) \\
\sin \left(\delta_{1}\right) & \cos \left(\delta_{1}\right)
\end{array}\right] \cdot\left[\begin{array}{c}
v_{X} \\
v_{Y 1}^{v e h}
\end{array}\right] } \\
\alpha_{1}= & \arctan \left(\frac{v_{Y 1}}{v_{X 1}}\right) \\
& \text { where } \frac{v_{Y 1}}{v_{X 1}}=-\frac{v_{Y}}{v_{X}}-\frac{\dot{\psi} \cdot l_{1}}{v_{X}}+\tan \left(\delta_{1}\right)
\end{aligned}
$$

In case of the rear virtual axle if it is not steered then the tire's plane is parallel to the vehicle's longitudinal plane, so the virtual axle's/tire's $\alpha_{2}$ sideslip angle is defined by $(12)$. For a steered front tyre (13) shows how longitudinal $v_{X 1}$ and lateral $v_{Y 1}$ velocities in the plane of the rotated front tyre could be calculated and (14) shows with some simplification and reordering how the front virtual axle's/tire's $\alpha_{1}$ sideslip angle could be calculated in the tire's direction $-\delta_{1}$ is the steered wheel angle.

$$
\begin{aligned}
a_{Y} \cdot m & =F_{Y 1} \cdot \cos \left(\delta_{1}\right)+F_{Y 2} \\
\ddot{\psi} \cdot I_{Z} & =F_{Y 1} \cdot \cos \left(\delta_{1}\right) \cdot l_{1}-F_{Y 2} \cdot l_{2} \\
\dot{x} & =A \cdot x+B \cdot u, \text { where } \\
A & =\left[\begin{array}{cc}
-\frac{c_{2}+c_{1}}{m \cdot v_{X}} & \frac{c_{2} \cdot l_{2}-c_{1} \cdot l_{1}}{m \cdot v_{X}^{2}}-1 \\
\frac{c_{2} \cdot l_{2}-c_{1} \cdot l_{1}}{I_{Z}} & -\frac{c_{2} \cdot l_{2}^{2}+c_{1} \cdot l_{1}^{2}}{I_{Z} \cdot v}
\end{array}\right] \\
B & =\left[\begin{array}{c}
\frac{c_{1}}{m \cdot v} \\
\frac{l_{1} \cdot c_{1}}{I}
\end{array}\right], \quad x=\left[\begin{array}{c}
\beta \\
\dot{\psi}
\end{array}\right], \quad \dot{x}=\left[\begin{array}{c}
\dot{\beta} \\
\ddot{\psi}
\end{array}\right] .
\end{aligned}
$$

The two lateral forces results $a_{Y}$ lateral acceleration 15 and $d^{2} \psi / d t^{2}$ yaw rotational acceleration $(16)$ with respect to $m$ mass and $I_{Z}$ vertical inertia. In case of the front lateral force steering direction should be considered as it can be seen. From these (17) shows the final linearized state space representation form - the state vector contains $\beta$ vehicle sideslip angle and $d \psi / d t$ yaw rate. As it could be seen the basic vehicle states are the vehicle frame's angular deviation with respect to its velocity and rotation velocity.

\section{Sideslip estimation}

As it was mentioned our aim is to develop a good enough vehicle sideslip estimation method. That could be used for a lot of new areas - for even a lane keeping function [4]. Without the definition of our method's operation one thing should be noticed: it must work outside of the linear range, too. In (17) linearized functions could be seen, it means that the cosine function's value is 1 , and the sine and tangent functions' values are their input angle in radians. Regarding these three functions the cosine's accuracy is the worst, $1 \% / 10 \%$ errors are resulted at $8.1^{\circ} / 26^{\circ}$. The same for sine is $14^{\circ} / 45^{\circ}$ and for tangent is $10^{\circ} / 30^{\circ}$. A typical lateral tyre force characteristic's maximum point is about $10-20 \%\left(5.7^{\circ}-11^{\circ}\right)[12]$ and after that it will be strongly non-linear [14] - Figure 1. In the figure you can see some numbers: number 1 marks a real lateral characteristic like shape, number 2 is a linear characteristic for the beginning of the shape, number 3 is a maximized case of the previous and number 4 is a locally linearized case.

In this way linearization works great if the (12) and 14

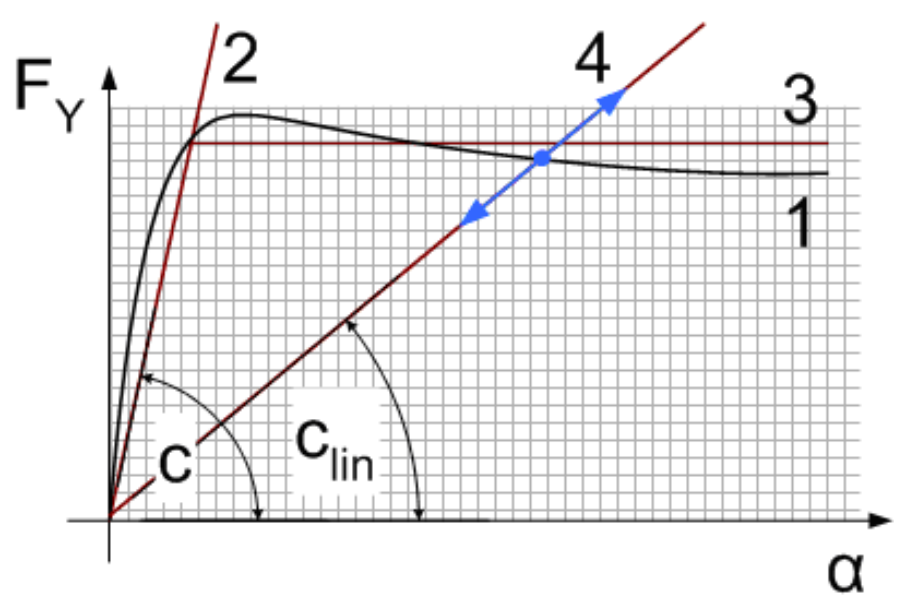

Fig. 1. Real and approximated lateral tyre characteristics

tyre sideslip angles nearly stay in the first linear zone (un$\operatorname{der} 5.7^{\circ}-11^{\circ}$ ) and the steered wheel angle stays under $14^{\circ} / 45^{\circ}$ commercial vehicles have more steerable wheels as passenger cars, but even their wheels couldn't be steered more than $40^{\circ}$ $45^{\circ}$ [9].Sideslip estimation's point is to estimate mainly nonlinear effects' results, which are the increased sideslip angles, so our method doesn't use simplified trigonometric functions. As it was mentioned our aim is to avoid any previously defined possibly varying information, too. It resulted that only Figure 1's number $4 c_{\text {lin }}$ linearized cornering stiffness parameter was suitable for us as tyre characteristic description. With this so called locally linearization the possibility of using of (17)'s linear state system could be held and for this the estimation method also outputs estimated cornering stiffness parameters. Fig. 2 shows a nonlinear maneuver's measured results (so these are not simulated results). The surface was wet plastic with less than 0.2 adhesion coefficient. Measurement errors could be seen in case of lateral acceleration - the frame roll influences accelerometer. It could be seen that the vehicle behaviour wasn't linear, significant drifts were realized, but the estimation method was capable to follow the vehicle sideslip angle.

\section{Vehicle stability improvement with sideslip estima- tion}

\subsection{Vehicle sideslip based directional control}

The main idea of vehicle sideslip based directional control is that it is a classical reference model free method, whose only condition is to keep vehicle sideslip angle's magnitude under a given limit that is about $3^{\circ}$. Figure 3 shows the contour of vehicle sideslip angles and lateral acceleration in case of a stable vehicle (the used tyre model for this calculation was nonlinear) - see later what is called stable vehicle in our case. There are two criteria of stable behaviour (which is not to be confused with stable vehicle) in this case: the first one is that vehicle sideslip angle's magnitude should be under $0.05 \mathrm{rad}$ (area outside of this limit is marked with yellow). The second one is that lateral acceleration should be under $4 \mathrm{~m} / \mathrm{s}^{2}$ (area outside of this limit is marked with red). If neither of the requirements is fulfilled then 

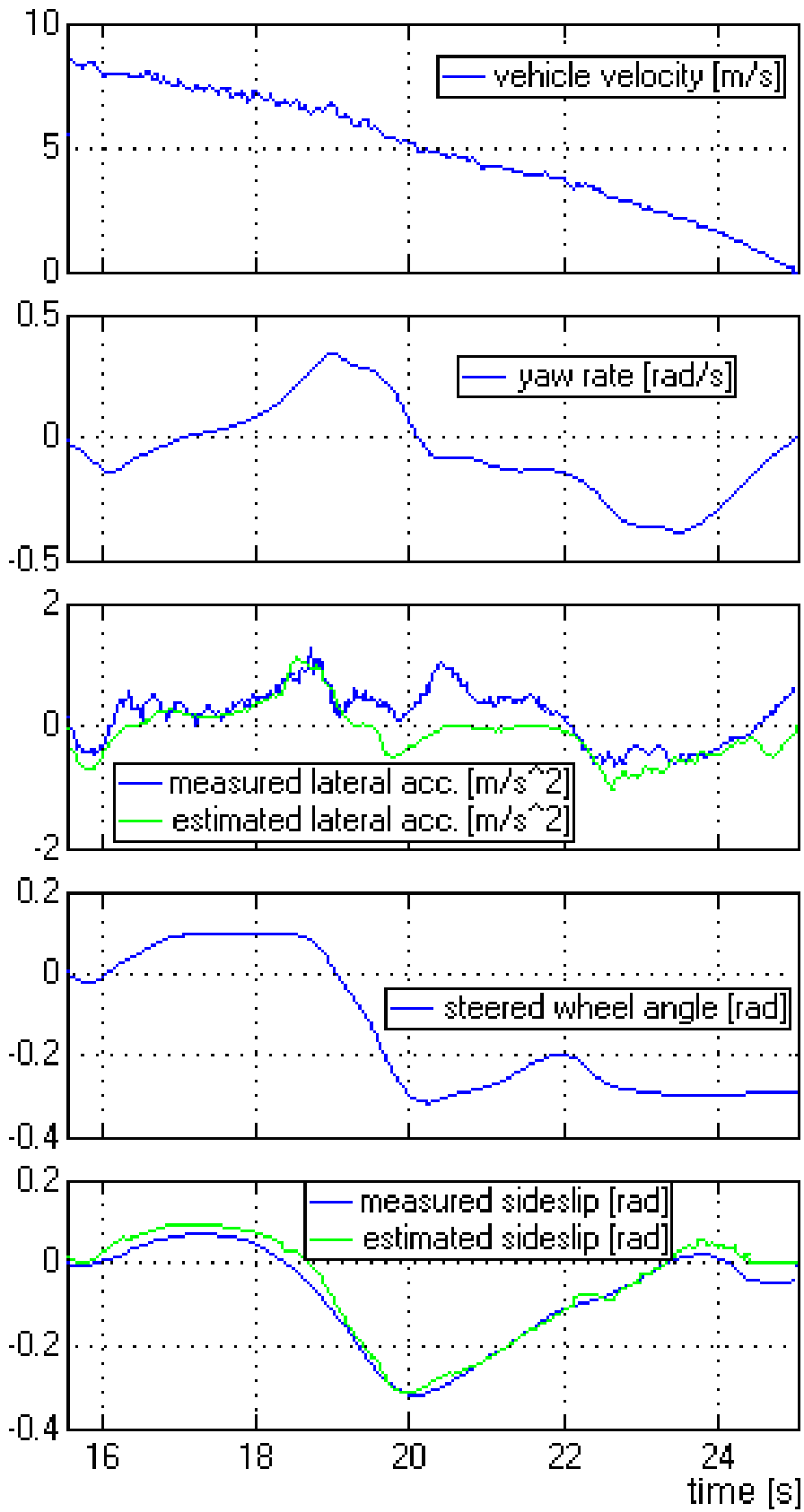

Fig. 2. Sideslip estimation results

it is marked with orange in the figure. These criteria's limits could be changed, the point is that with the vehicle sideslip limitation controllability could be held and with the lateral acceleration limitation vehicle rollover situations could be predicted. Suppose that an external control method doesn't let the vehicle to reach more than $4 \mathrm{~m} / \mathrm{s}^{2}$ lateral acceleration and consider only stationary cases, thus only at low velocities is the absolute vehicle sideslip over $0.05 \mathrm{rad}$. But these cases are typically parking maneuvers or low speed turnings where the reached sideslip angle is near to the Ackermann angle - these cases shouldn't be controlled. Figure 3 shows a left turning; positive steering angles mean counter clockwise steering wheel rotation (the steering angle in the figure marks steered wheel angle). At low velocities the vehicle realizes positive sideslip angles and negative sideslip angles are reached at high velocities. In case of positive vehicle sideslip the steering centre of the vehicle is behind the $\mathrm{CoG}$ and with acceleration when the vehicle sideslip starts to reach negative values the steering centre is going to the front of the vehicle.

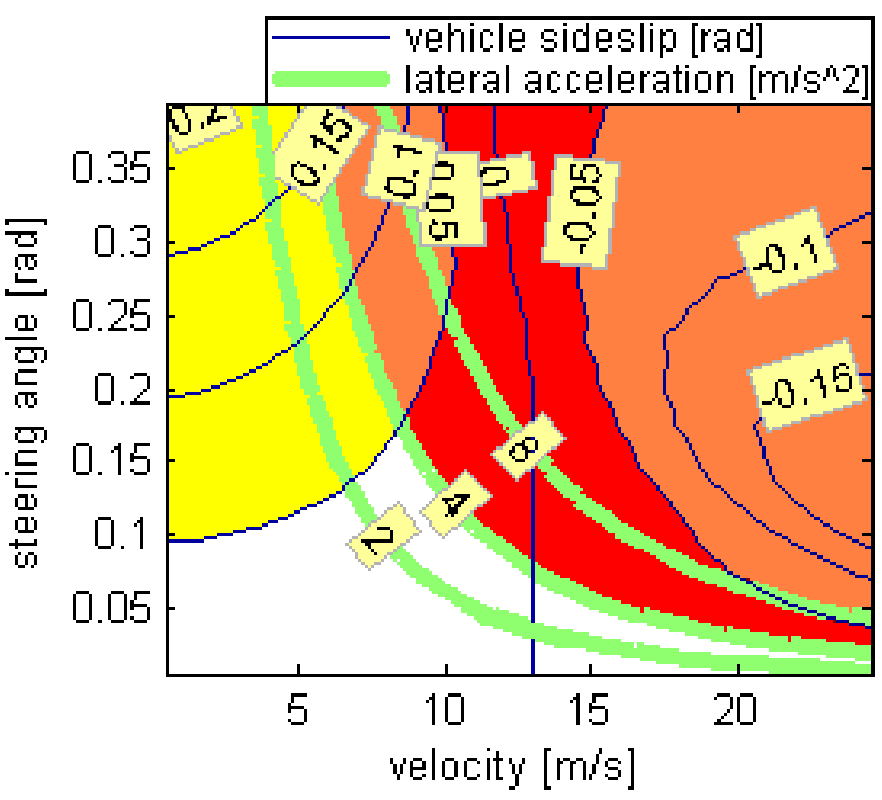

Fig. 3. Stationary vehicle sideslip and lateral acceleration map for a stable vehicle

On the basis of above mentioned things we created an always stable adaptive reference model (ARM) that doesn't require any tuning, it only uses the cornering stiffness parameter estimation's results and defines always the reference lateral acceleration (via reference yaw rate) and the maximum allowed vehicle sideslip limits, whose aim is to ensure comfortable low velocity maneuvers, too. First of all a mathematically stable vehicle model had to be calculated. For the ARM we used (17)'s linear bicycle model and as stability requirement we declared a BIBO system that responds to bounded input signals with bounded output signals. For a linear state space representation BIBO stability could be defined by the state matrix's $\lambda$ poles [2] $[18)-\mathbf{I}$ is an identity matrix with suitable dimensions. It could be calculated with a quadratic equation's solving, and the $\lambda$ roots (poles) must be in the imaginary scale's left side - the real part must be negative. The quadratic solving formula's non square component will be always negative, so a relation condition could be set up to ensure that the roots are negative. With some further calculations, reordering and simplifications 19 will be given as condition of stability and from this 20 ensures a way how (17)'s linear reference model could be always stable. The cornering stiffness parameter estimation results two signals, $c_{1}$ and $c_{2}$ and with some processing by (21) the condition of 20 ) could be easily carried out.

$$
\begin{aligned}
& \operatorname{det}(\lambda \cdot I-A)=0 \\
& \left|\begin{array}{cc}
\lambda+\frac{c_{2}+c_{1}}{m \cdot v_{X}} & \frac{c_{1} \cdot l_{1}-c_{2} \cdot l_{2}}{m \cdot v_{X}^{2}}+1 \\
\frac{c_{1} \cdot l_{1}-c_{2} \cdot l_{2}}{I_{Z}} & \lambda+\frac{c_{2} \cdot l_{2}^{2}+c_{1} \cdot l_{1}^{2}}{I_{Z} \cdot v}
\end{array}\right|=0 \\
& v_{X}^{2}<\frac{c_{1} \cdot c_{2} \cdot\left(l_{1}+l_{2}\right)^{2}}{m \cdot\left(c_{1} \cdot l_{1}-c_{2} \cdot l_{2}\right)}
\end{aligned}
$$




$$
\begin{gathered}
c_{1} \cdot l_{1}=c_{2} \cdot l_{2} \\
{\left[\begin{array}{c}
c_{1}^{s t a b} \\
c_{2}^{\text {stab }}
\end{array}\right]=\max \left(c_{1} \cdot l_{1}, c_{2} \cdot l_{2}\right) \cdot\left[\begin{array}{c}
\frac{1}{l_{1}} \\
\frac{1}{l_{2}}
\end{array}\right]}
\end{gathered}
$$

But even with this always stable linear state space ARM, it couldn't be guaranteed that the lateral acceleration and vehicle sideslip reference values will be between the limits. For former a double circuit ARM technique is used: the first ARM calculates lateral acceleration as well and if it is over the limit then simply an approximately vehicle speed will be calculated and this will be the second ARM's input velocity (because the first ARM's lateral acceleration output is continuous and the velocity limitation function is also continuous, the second ARM's velocity input will be also continuous). The calculation considers the relative value of lateral acceleration exceeding and decreases the velocity with square proportion. In this way instead of saturation of ARM outputs the second ARM could have a continuous state whose lateral acceleration output is under the limit (with a good approximation) but at the same time vehicle sideslip and yaw rate dynamics are continuous. Furthermore the second ARM's vehicle sideslip output is able to expand vehicle sideslip limit values but only in the steering wheel angle's sign's direction as it can be seen in Figure 4. It shows a left turn with $20 \mathrm{~km} / \mathrm{h}$ vehicle speed. During the maneuver the steered wheel angle is increased until the $50^{\text {th }}$ second and then it is decreased. The maximal steered wheel angle is $35^{\circ}(=0.61 \mathrm{rad})$ and the reached vehicle sideslip is about $0.22 \mathrm{rad}\left(=12.6^{\circ}\right)$ that is near to the Ackermann angle at this steered wheel angle $\left(16^{\circ}=0.28 \mathrm{rad}\right)$. During the left turn positive vehicle sideslip is allowed to avoid unnecessary interventions and to ensure comfortable maneuvering ability.

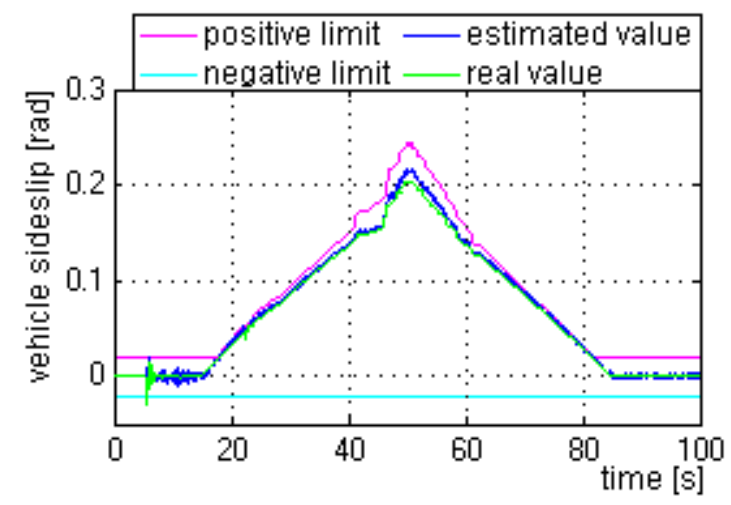

Fig. 4. Vehicle sideslip angle limits during a slow left turn maneuver

\subsection{Vehicle state prediction}

For state prediction we searched a suitable input signal prediction method. As input only $\delta_{1}$ steering angle is considered, $v_{X}$ vehicle velocity is a parameter. Regarding steered wheel angle a simple assumption is accepted for our system: the steered wheel angle is a 22) sinusoidal function, whose $\Phi$ phase rotation velocity and amp amplitude should be estimated. For the amplitude calculation first an actual $\varphi$ phase should be calculated (23), then previous steps' measured steered wheel angles result an estimated $\Phi$ steering angle phase rotation velocity with consideration of $\Delta t$ sampling time (24). The calculated sinusoidal steering angle will be a linear system's input (based on (17) again). For this linear system as parameters current vehicle velocity and 21) corrected cornering stiffness parameters are considered. Some cornering stiffness parameter correction is necessary to ensure the stability of the calculations.

$$
\begin{gathered}
\delta_{1}\left\{\begin{array}{l}
\delta_{1}=a m p \cdot \cos (\phi) \\
\dot{\delta}_{1}=-a m p \cdot \sin (\phi) \\
\delta_{1}^{-1}=a m p \cdot \cos (\phi-\Delta \phi) \\
\delta_{1}^{-2}=a m p \cdot \cos (\phi-2 \cdot \Delta \phi) \\
\Delta \phi=\Delta t \cdot \varphi
\end{array}\right. \\
\phi=\arctan \left(-\frac{\dot{\delta}_{1}}{\delta_{1}}\right) \quad a m p=\frac{\delta_{1}}{\cos (\phi)} \\
\Delta t \cdot \varphi=\arccos \left(\frac{\delta_{1}^{-1}}{a m p}\right)-\arccos \left(\frac{\delta_{1}^{-2}}{a m p}\right)
\end{gathered}
$$

After the input signal's prediction with the using of current vehicle velocity and corrected cornering stiffness parameters only further calculations are needed. Thus 25 and 26 show how vehicle sideslip and yaw rate transmission functions could be separately calculated. As 27) shows these $F(s)$ functions could be rearranged in general, and with using of the $G(s)$ sine input function's Laplace transformed shape (28) shows the transmission functions' Laplace transformed shape. To transform these equations into time domain convolution multiplying and later partial integration are needed and then 29]'s $Y(t)$ result will have trigonometric and exponential components. With neglecting of exponential components a pure trigonometric function will be given and the maximum places could be calculated with some derivation.

$$
\begin{aligned}
& {\left[\begin{array}{l}
\beta \\
\dot{\psi}
\end{array}\right] \cdot s=\left[\begin{array}{ll}
A_{11} & A_{21} \\
A_{12} & A_{22}
\end{array}\right] \cdot\left[\begin{array}{l}
\beta \\
\dot{\psi}
\end{array}\right]+\left[\begin{array}{l}
B_{11} \\
B_{12}
\end{array}\right] \cdot \delta_{1}} \\
& {\left[\begin{array}{c}
\beta \\
\dot{\psi}
\end{array}\right]=\frac{\left[\begin{array}{ll}
a_{\beta} & b_{\beta} \\
a_{\dot{\psi}} & b_{\dot{\psi}}
\end{array}\right] \cdot\left[\begin{array}{c}
s \\
1
\end{array}\right]}{s^{2} \cdot c+s \cdot d+e} \cdot \delta_{1}} \\
& \text { where }\left\{\begin{array}{c}
a_{\beta}=B_{11} \quad a_{\dot{\psi}}=B_{12} \\
b_{\beta}=B_{12} \cdot A_{21}-A_{22} \cdot B_{11} \\
b_{\beta}=B_{11} \cdot A_{12}-A_{11} \cdot B_{12} \\
c=1 \quad d=-A_{11}-A_{22} \\
e=A_{11} \cdot A_{22}-A_{21} \cdot A_{12}
\end{array}\right. \\
& F(s)=\frac{s \cdot a+b}{s^{2} \cdot c+s \cdot d+e}=\frac{1}{c} \cdot \frac{s \cdot a+b}{\left(s+T_{1}\right) \cdot\left(s+T_{2}\right)} \\
& F(s) \cdot G(s)=\frac{1}{c} \cdot \frac{s \cdot a+b}{\left(s+T_{1}\right) \cdot\left(s+T_{2}\right)} \cdot \frac{\varphi}{s^{2}+\varphi^{2}}
\end{aligned}
$$




$$
\begin{aligned}
& Y(t)=\frac{\left[\begin{array}{lll}
1 & \varphi & \varphi
\end{array}\right] \cdot v \cdot\left[\begin{array}{l}
\frac{a \cdot T_{1}-b}{\varphi^{2}+T_{1}^{2}} \\
-\frac{a \cdot T_{2}-b}{\varphi^{2}+T_{2}^{2}}
\end{array}\right]}{c \cdot\left(T_{1}-T_{2}\right)} \\
& \text { where } \\
& \left\{v=\left[\begin{array}{ll}
T_{1} \cdot \sin (\varphi \cdot t) & T_{2} \cdot \sin (\varphi \cdot t) \\
-\cos (\varphi \cdot t) & -\cos (\varphi \cdot t) \\
\exp \left(-T_{1} \cdot t\right) & \exp \left(-T_{2} \cdot t\right)
\end{array}\right]\right.
\end{aligned}
$$

For lateral acceleration yaw rate and vehicle sideslip rate is necessary. Latter's transfer function is not given by us above but with simple derivation it could analytically calculated from 29. and with further derivation the maximum places can be found again. Figure 5 shows a prediction method during an accelerating sine steering wheel input test. The prediction considers the stable vehicle's 21) cornering stiffness parameters - that's why the prediction is higher than the real values. With some cornering stiffness parameter tuning different prediction levels could be reached.

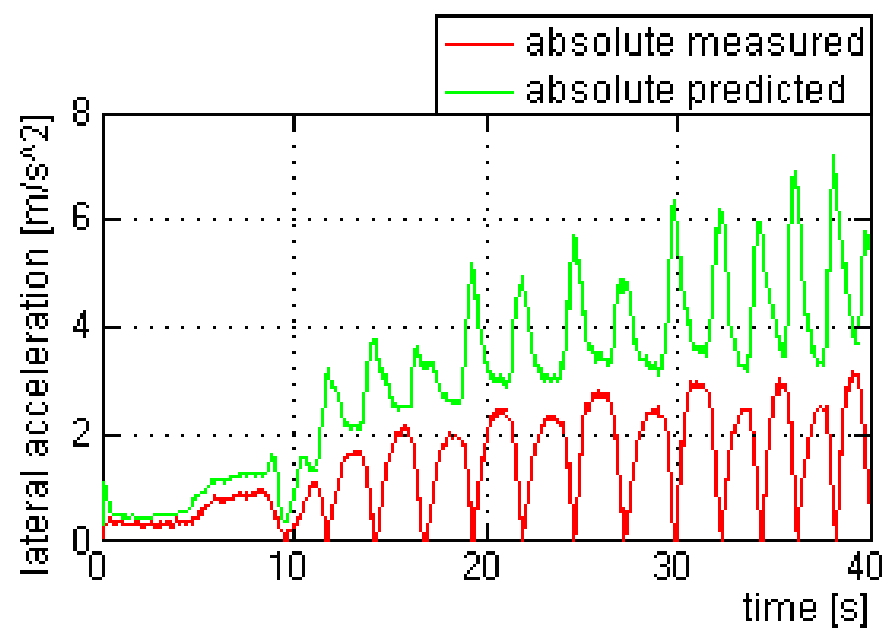

Fig. 5. Sine steering wheel's predicted lateral acceleration

As it can be seen in the figure the predicted values are the highest at the beginning of the sine waves - as the driver starts to reach the highest steering angle he slows down the steering velocity. Furthermore it could also be seen that the prediction doesn't fall under a given level which is about the reached maximum lateral accelerations. This could result a predictive engine torque reduction [6] and small braking on the better grip having axle. Yaw rate and sideslip angle prediction could be done in the same way.

\subsection{Active servo motor}

In contrast to steer-by-wire ( $\mathrm{SbW}$ ) conceptions in case of an active servo motor the steering wheel and the steered wheels are in mechanical connection - like in case of an everyday steering system. It sounds natural for the first sight. But in case of a SbW system two separated steering angles could be realized: one shows the driver's demand and with the other, which controls the steered wheels, the vehicle state defined by this demand could be reached. There is no separated driver's demand regarding the active servo motor; there is only the mentioned direct link between the driver and the steered wheels. Thus the driver should be influenced - together with the steered wheels and this results an interesting situation: first, there is a steering wheel position that results in a target trajectory. Second, the vehicle has some deviation from this target trajectory and this will cause an active servo torque that tries to help the driver. Third, this active torque will result in a modified steering wheel position and the point here is: this modified position must not cause an increased servo torque. An example: if a vehicle is understeered then for a given steering wheel position (as a trajectory target defining marker) increased steered wheel position would be necessary. But these angles are connected together so understeering would result more steering angles that would result more understeering that would results more steering angle and so on.

As we imagine this function, it could teach the driver what the vehicle's actual limits are. In case of lateral acceleration control or yaw rate control only returning torques could be used. It means that the servo torque mustn't encourage the driver to steer more - in any stable cases (oversteering or understeering) steering angle increasing could cause more instability. If the vehicle is understeered then returning moment suggests back steering or if the vehicle is oversteered then to keep vehicle stability back steering also would be necessary.

When vehicle sideslip control is necessary then the situation differs. In these cases there is an unambiguous aim that is not defined by the steering wheel angle: keep vehicle sideslip angle under the defined limit. So steering wheel angle is secondary and servo torque is controlled by vehicle sideslip limitation efforts. In a situation like this the active servo torque tries to stabilize the vehicle regardless the steering angle otherwise the vehicle would be uncontrollable. Figure 6 shows a sine steering wheel input with accelerating and running into low adhesion zone with simulation technique. Latter begins after the $55^{\text {th }}$ second. Before this point the vehicle accelerates and it will be more and more understeered - the active servo logic tries to moderate the driver's steering angle (as it can be seen in the servo torque graph of the figure) and as the velocity and the understeered behaviour increase the active servo torque also increases. After the $55^{\text {th }}$ second a low adhesion zone is reached - the driven rear wheels lose their lateral grip and significant skids start to be realized. These skids are controlled in the right time, the maximum reached vehicle sideslip angle is under $0.1 \mathrm{rad}\left(=5.7^{\circ}\right)$. The skid control is performed by servo torque control change and differential brake torques. Previous could be seen in the graph: when a skid is started then the active servo torque's shape isn't smooth anymore, it is harsh and stronger than before. Furthermore differential brake torque is realized - as it can be seen not in only one side. It is important to notice that commercial vehicles' steered axle braking is different from passenger vehicles. Latter stay stable if one of the steered wheels is stronger braked. In contrast with this commercial vehicles gain the differential 
braking's effect and steers themselves into the stronger braking's direction, so an interesting steer-by-brake mechanism could be realized. In Figure 6's case it can be seen that when a skid happens then a returning servo torque is realized and at the same time a brake force is realized in the same direction. When the vehicle sideslip will be again between the limits then additive differential brake forces will be stopped and smooth yaw rate control based active servo torque is returned. It should also be noticed that in the figure yaw rate and lateral acceleration are between limits. It means that yaw rate based directional control could have intervened only later. Later intervention would have resulted stronger braking and it would have resulted a significant velocity loss - so with more control energy less efficiency could have been reached.

\section{Summary}

The improvement of active vehicle safety is a primary target of automotive developers. Electronic stability program's next generation may consider vehicle sideslip angle. With the using of a technology like this several advantages could be reached: first the recently used brake intervention units' control could be improved. In case of yaw rate based directional control the intervention is effective, but not as effective as it could be. If the aim is the highest level of vehicle stability then vehicle sideslip based directional control recognizes earlier dangerous situations and controls with less energy. If the aim is the latest intervention that is possible then vehicle sideslip control could also recognize this moment, which is often after the currently used yaw rate based control's intervention. Moreover these recognitions depend on vehicle state (e.g. payload) and the control logic doesn't try to keep always the same reference.

Predictions could also be made with the knowing of vehicle sideslip angle. These predictions could result earlier engine torque reductions or preventive small brakings. At the beginning of a maneuver a final highest lateral acceleration or vehicle sideslip could be estimated. With this information the safest stability control could be realized: the avoidance of dangerous situations.

Active servo motor has the same effect. If the driver gets active feedback about the vehicle state then he/she can modify the vehicle trajectory demand to reach a safer driving. Even with an active servo torque instead of a steer-by-wire solution vehicle stability could be held: when a skid happens the aim is not defined by the steering wheel angle. In these cases the aim is to keep vehicle sideslip under the limit value, so active servo torque has bigger freedom as in case of yaw control.

Anyway, with a vehicle sideslip estimation better vehicle stability control could be reached. And in the end better vehicle stability results saved human life.
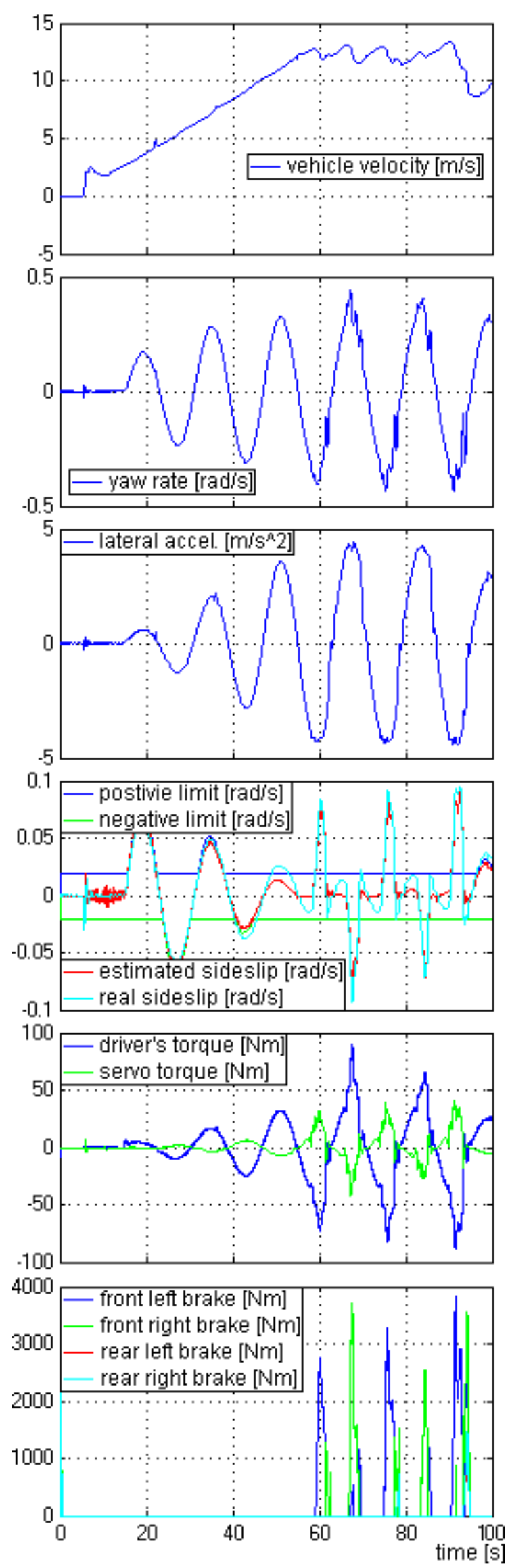

Fig. 6. Running into low-mu zone 


\section{Appendix}

\begin{tabular}{|c|c|c|}
\hline Notation & Meaning & Unit \\
\hline$v$ & Absolute vehicle velocity & $\mathrm{m} / \mathrm{s}$ \\
\hline$\theta$ & Vehicle velocity direction & $\mathrm{rad}$ \\
\hline$i$ & Plane base vector 1 & - \\
\hline$j$ & Plane base vector 2 & - \\
\hline$e_{T}$ & Tangential velocity base vector & - \\
\hline$e_{N}$ & Normal velocity base vector & - \\
\hline$\psi$ & Yaw angle & $\mathrm{rad}$ \\
\hline$\theta$ & Velocity direction wrt. $i$ & $\mathrm{rad}$ \\
\hline$\beta$ & Vehicle sideslip angle & $\mathrm{rad}$ \\
\hline$v_{X}$ & Longitudinal vehicle velocity & $\mathrm{m} / \mathrm{s}$ \\
\hline$v_{Y}$ & Lateral vehicle velocity & $\mathrm{m} / \mathrm{s}$ \\
\hline$a_{X}$ & Longitudinal vehicle acceleration & $\mathrm{m} / \mathrm{s}^{2}$ \\
\hline$a_{Y}$ & Lateral vehicle acceleration & $\mathrm{m} / \mathrm{s}^{2}$ \\
\hline$F_{Y}$ & Lateral tyre force & $\mathrm{N}$ \\
\hline$\alpha$ & Tyre sideslip angle & $\mathrm{rad}$ \\
\hline$c$ & Tyre cornering stiffness coeff. & $\mathrm{N} / \mathrm{rad}$ \\
\hline$v_{Y 1}^{v e h}$ & Lateral $1^{\text {st }}$ axle vel. in veh. sys. & $\mathrm{m} / \mathrm{s}$ \\
\hline$v_{Y 2}^{v e h}$ & Lateral $2^{\text {nd }}$ axle vel. in veh. sys. & $\mathrm{m} / \mathrm{s}$ \\
\hline$l_{1}$ & Front axle distance from $\mathrm{CoG}$ & $\mathrm{m}$ \\
\hline$l_{2}$ & Rear axle distance from $\mathrm{CoG}$ & $\mathrm{m}$ \\
\hline$\alpha_{2}$ & Rear axle sideslip angle & $\mathrm{rad}$ \\
\hline$v_{X 1}$ & Long. $1^{\text {st }}$ axle vel. in. tyre sys. & $\mathrm{m} / \mathrm{s}$ \\
\hline$v_{Y 1}$ & Lateral $1^{\text {st }}$ axle vel. in. tyre sys. & $\mathrm{m} / \mathrm{s}$ \\
\hline$\alpha_{1}$ & Front axle sideslip angle & $\mathrm{rad}$ \\
\hline$\delta_{1}$ & Front axle steered wheel angle & $\mathrm{rad}$ \\
\hline$d^{2} \psi / d t^{2}$ & Yaw acceleration & $\mathrm{rad} / \mathrm{s}^{2}$ \\
\hline$m$ & Vehicle mass & $\mathrm{kg}$ \\
\hline$I_{Z}$ & Vehicle vertical inertia & $\mathrm{kg} / \mathrm{m}^{2}$ \\
\hline$d \psi / d t$ & Yaw rate & $\mathrm{rad} / \mathrm{s}$ \\
\hline$c_{\text {lin }}$ & Linearized corner. stiff. coeff. & $\mathrm{N} / \mathrm{rad}$ \\
\hline$I$ & Identity matrix & - \\
\hline$\lambda$ & Transfer function's pole & - \\
\hline$c_{1}$ & Front axle corner. stiff. coeff. & $\mathrm{N} / \mathrm{rad}$ \\
\hline$c_{2}$ & Rear axle corner. stiff. coeff. & $\mathrm{N} / \mathrm{rad}$ \\
\hline$\Phi$ & Phase rotation velocity & $\mathrm{rad} / \mathrm{s}$ \\
\hline amp & Amplitude & $\mathrm{rad}$ \\
\hline$\varphi$ & Phase & $\mathrm{rad}$ \\
\hline$\Delta t$ & Sample time & $\mathrm{s}$ \\
\hline$F(s)$ & Transfer function & - \\
\hline$G(s)$ & Excitation function & - \\
\hline$Y(t)$ & Transition function & - \\
\hline
\end{tabular}

\section{References}

1 Ardema Mark D, Newton-Euler Dynamics, Springer Science+Business Media, Inc.; USA, 2005.

2 Bokor J, Gáspár P, Irányítástechnikai jármüdinamikai alkalmazásokkal, Typotex kft; Budapest, 2008.

3 Farmer Charles M, Effects of Electronic Stability Control on Fatal Crash Risk, Insurance Institute for Highway Safety; Arlington, Vancouver, USA, 2010.

4 Hankovszki Z, Kovács R, Palkovics L, Lane keeping for heavy duty commercial vehicles, 22nd International Symposium on Dynamics of Vehicles on Roads and Tracks, (2011).

5 Insurance Institute for Highway Safety, Status Report, 41(5), (2006).

6 Lakatos I, Effect timing on the effiency and exhaust of four-stroke, uncharged SOHC Otto-engines, microCAD International Scientific Conference, (2004).

7 Lakatos I, Effect of valve timing on exhaust emission, 8th International Conference on Heat Engines and Environmental Protection, (2007).

8 Leenen Roel, Schouten Hanno, Virtual sensors for advanced vehicle stability control, 10th International Symposium on Advanced Vehicle Control, (2010).

9 MAN Nutzfahrzeuge, Grundlagen der Nutzfahrzeugtechnik: Basiswissen Lkw und Bus; München, 2009.

10 Németh B, Gáspár P, Considering predicted road conditions in platoon control design, Periodica Polytechnica Transportation Engineering, 39(2), (2011), DOI 10.3311/pp.tr.2011-2.04

11 Palkovics L, Jármúirányítás és menetstabilizálás, Magyar Tudomány, (2005), 561 .

12 Pacejka Hans B, Tyre and Vehicle Dynamics, 2006. Second Edition.

13 Robert Bosch GmbH, Driving Stability Systems; München, 2005.

14 Szabó B, Vehicle test based validation of a tire brush model using an optical velocity sensor, Periodica Polytechnica Transportation Engineering, 40, (2012), DOI 10.3311/pp.tr.2012-1.06

15 Trencséni B, Palkovics L, Driveline torque observer for heavy duty vehicles, Periodica Polytechnica Transportation Engineering, 39(2), (2011), DOI 10.3311/pp.tr.2011-2.08

16 United Nations Economic Commission for Europe, 2011. Regulation 13, 11 Series of Amendments.

17 Zomotor Á, Reimpell J, Fahrwerktechnik, Fahrverhalten, Vogel Verlag und Druck; Germany, 1991. 